\title{
Improving accuracy of overhanging structures for selective laser melting through reliability characterization of single track formation on thick powder beds
}

\author{
Mohanty, Sankhya; Hattel, Jesper Henri
}

Published in:

Proceedings of SPIE

Link to article, DOI:

$10.1117 / 12.2212621$

Publication date:

2016

Document Version

Publisher's PDF, also known as Version of record

Link back to DTU Orbit

Citation (APA):

Mohanty, S., \& Hattel, J. H. (2016). Improving accuracy of overhanging structures for selective laser melting through reliability characterization of single track formation on thick powder beds. In B. Gu, H. Helvajian, \& A. Piqué (Eds.), Proceedings of SPIE (Vol. 9738). [97381B] SPIE - International Society for Optical Engineering. Proceedings of SPIE - The International Society for Optical Engineering https://doi.org/10.1117/12.2212621

\section{General rights}

Copyright and moral rights for the publications made accessible in the public portal are retained by the authors and/or other copyright owners and it is a condition of accessing publications that users recognise and abide by the legal requirements associated with these rights.

- Users may download and print one copy of any publication from the public portal for the purpose of private study or research.

- You may not further distribute the material or use it for any profit-making activity or commercial gain

- You may freely distribute the URL identifying the publication in the public portal 


\title{
Improving accuracy of overhanging structures for selective laser melting through reliability characterization of single track formation on thick powder beds
}

\author{
Sankhya Mohanty, Jesper Henri Hattel \\ Dept. of Mechanical Engineering, Technical University of Denmark, Produktionstorvet, \\ Kongens Lyngby, Denmark 2800
}

\begin{abstract}
Repeatability and reproducibility of parts produced by selective laser melting is a standing issue, and coupled with a lack of standardized quality control presents a major hindrance towards maturing of selective laser melting as an industrial scale process. Consequently, numerical process modelling has been adopted towards improving the predictability of the outputs from the selective laser melting process. Establishing the reliability of the process, however, is still a challenge, especially in components having overhanging structures.

In this paper, a systematic approach towards establishing reliability of overhanging structure production by selective laser melting has been adopted. A calibrated, fast, multiscale thermal model is used to simulate the single track formation on a thick powder bed. Single tracks are manufactured on a thick powder bed using same processing parameters, but at different locations in a powder bed and in different laser scanning directions. The difference in melt track widths and depths captures the effect of changes in incident beam power distribution due to location and processing direction. The experimental results are used in combination with numerical model, and subjected to uncertainty and reliability analysis. Cumulative probability distribution functions obtained for melt track widths and depths are found to be coherent with observed experimental values. The technique is subsequently extended for reliability characterization of single layers produced on a thick powder bed without support structures, by determining cumulative probability distribution functions for average layer thickness, sample density and thermal homogeneity.
\end{abstract}

Keywords: selective laser melting, overhanging structures, single track formation, regression analysis, Monte Carlo simulations, reliability estimation, uncertainty characterization.

\section{INTRODUCTION}

In selective laser melting, most of the nearly 130 parameters [1] identified can be explained to have uncertainties associated with them, but with varied influence on the final outcomes of the SLM process. Thus, there is an increased effort towards developing procedures for in-process inspection and quality control including, but not limited to, thermal imaging [2] and ultrasonic [3] methods. Researchers have also attempted to establish reliability of the process by trying to characterize single scanning track formations using combination of statistical, numerical and experimental techniques [4] [5].

From a numerical process modelling viewpoint, till date, the focus has been limited to a few parameters e.g. the power of laser beam, the scan speed, FWHM of the laser beam, the porosity of powder bed, the average powder diameter, the chamber/environment temperature and the albedo of the powder particles. Of these, the power and scan speed have been found to have a low degree of associated uncertainty as they are often controlled parameters. The FWHM of laser beams have been known to vary during the processing due to thermal effects on the optical components of the selective laser melting [1]. The typical powders used in SLM are characterized by a normal distribution with respect to the powder diameter [6]. Additionally, the powder particles do not

Laser 3D Manufacturing III, edited by Bo Gu, Henry Helvajian, Alberto Piqué, Proc. of SPIE Vol. 9738, 97381B · C 2016 SPIE · CCC code: 0277-786X/16/\$18 · doi: 10.1117/12.2212621 
necessarily possess the same optical properties (albedo, extinction coefficients, etc.) as those reported in literature for bulk materials. The porosity is also significantly uncertain as no proper morphology/packing can be attributed to the powder bed. Typically the building chamber is kept at an elevated temperature during SLM process (as this has been found to have beneficial effects with regards to the mechanical properties and residual stress states of the manufactured products) through a combination of substrate heating [7] and gas flow [8]. However, SLM machines usually record the substrate temperature, and the actual local chamber temperature is unknown.

As these uncertainties in physical parameters are seldom transferred onto deterministic numerical process models, the prediction results from such models may not necessarily agree with the experimental observations. The presence of uncertainties being inherently accepted, the need to characterize the occurrence of these uncertainties is necessary. Thus, a methodology has to be established to ensure that the uncertainties in input parameters are accounted for and propagated when predicting outputs. Formal characterization of the aforementioned uncertainty associated with a process leads to the establishment of the process reliability. Thus, reliability of a process can be established by characterizing the effect of small uncertainties in inputs on the desired output of the process. The subject has been studied extensively for statistical systems and its usage in combination with complex physical models is also increasing. Specifically, the infeasibility of replicating exact conditions as needed by a simulation or model as well as those present during experimental runs, makes uncertainty characterization applicable to most engineering fields [9] [10] [11]. For a better understanding of the concepts used in this paper one might refer to the works of Saltelli et al [12] [13].

In this paper, the aforementioned concepts have been used to establish the reliability of the process, especially in producing components having overhanging structures. In this regard, an overhanging structure is envisioned as a combination of single tracks formed on a deep powder bed as opposed to the thin powder layer prevalent in most of the manufactured part. Thus, establishing the reliability of single tracks formed on a deep powder bed followed by the reliability of single layer formation can be construed as the first steps towards establishing the reliability of the process.

\section{SINGLE MELT TRACKS ON DEEP POWDER BED}

Laser power is one of the most commonly investigated parameters in experimental studies on SLM, often coupled with scanning speed [4]. A common theme of research, then, is to predict the effect of laser power (or energy density) on the melt track formation either through measurement of melt track width and depth or through in-process tracking of melt pool temperatures and dimensions [2]. The influence of location in the powder bed has also been studied, albeit from the perspective of temperature and gas flow in the chamber [8].

In this study, the former approach was adopted and laser powers at two different settings were used for experiments. Further, the combined effect of change in location of powder bed and the directionality of laser scanning was planned to be captured. For this purpose, single melt tracks were generated at different locations of the powder bed in different directions. The entire powder bed was divided into four regions corresponding to four quadrants with respect to the center of the powder bed. Several single melt tracks were generated in four different directions in each quadrant. The intention was to capture the elongation of incident laser beam spot (Figure 1) due to angular incidence in a region away from the center of the powder bed (where optical elements of the laser system are located). Further it was expected that the elongation of the beam would result in different melt track widths and depths depending on whether the direction of scanning was parallel or orthogonal to the direction of elongated axis.

The experiments were carried out at laser powers of $50 \mathrm{~W}$ and $120 \mathrm{~W}$ for Ti-6Al-4V. A scanning speed of $8.5 \mathrm{~mm} / \mathrm{s}$ was chosen for generating the melt tracks with a chamber temperature of $200^{\circ} \mathrm{C}$. The powder particles had a diameter distributed about $25 \mu \mathrm{m}$, and the powder bed had a layer depth of $5 \mathrm{~mm}$. Figure 2 shows a section of the build chamber with single melt tracks formed upon a deep powder bed. The single tracks formed by both the laser power values were then measured to find the molten-resolidified track width as shown in Figure 3 as well as the width of the sintered track. As both the molten-resolidified as well as the sintered areas are important in overhanging structures, the combined width would hereon be referred to as width of melt track in this paper. 

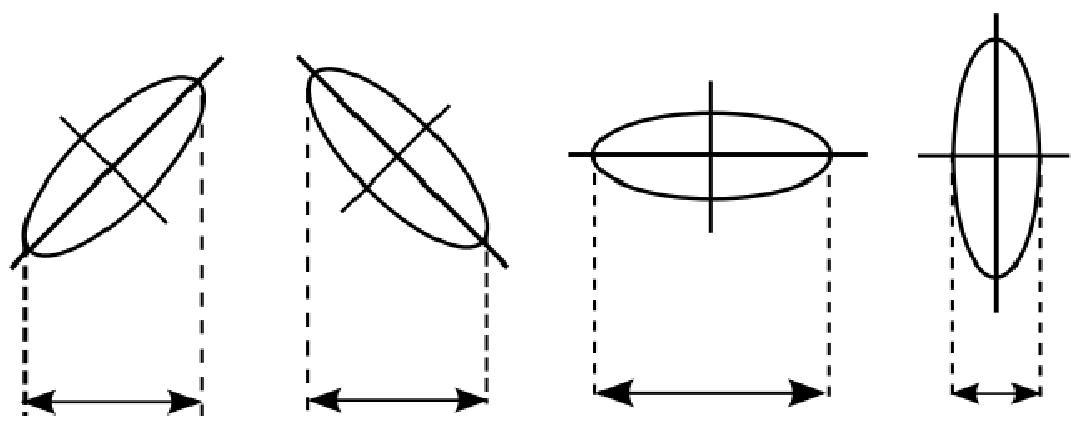

Figure 1 Elongation of laser spot upon angular incidence at regions away from center of powder bed

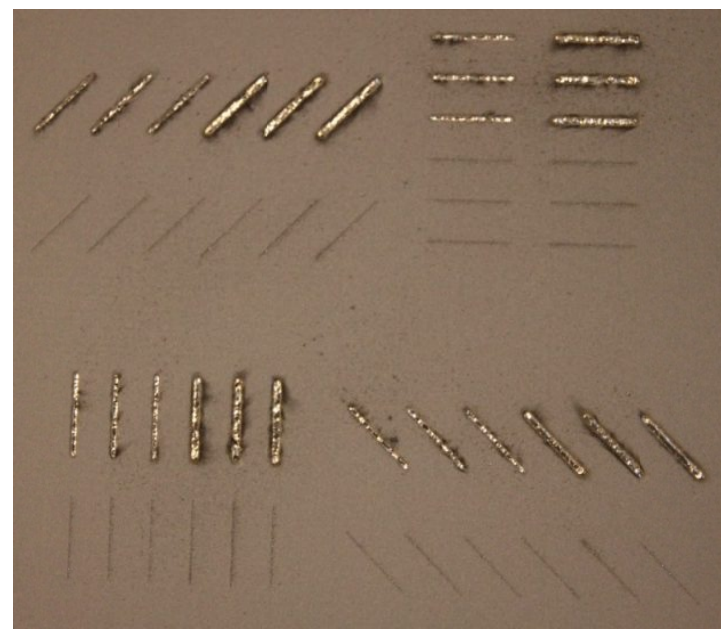

Figure 2 Single tracks in four different directions in a section of the powder bed

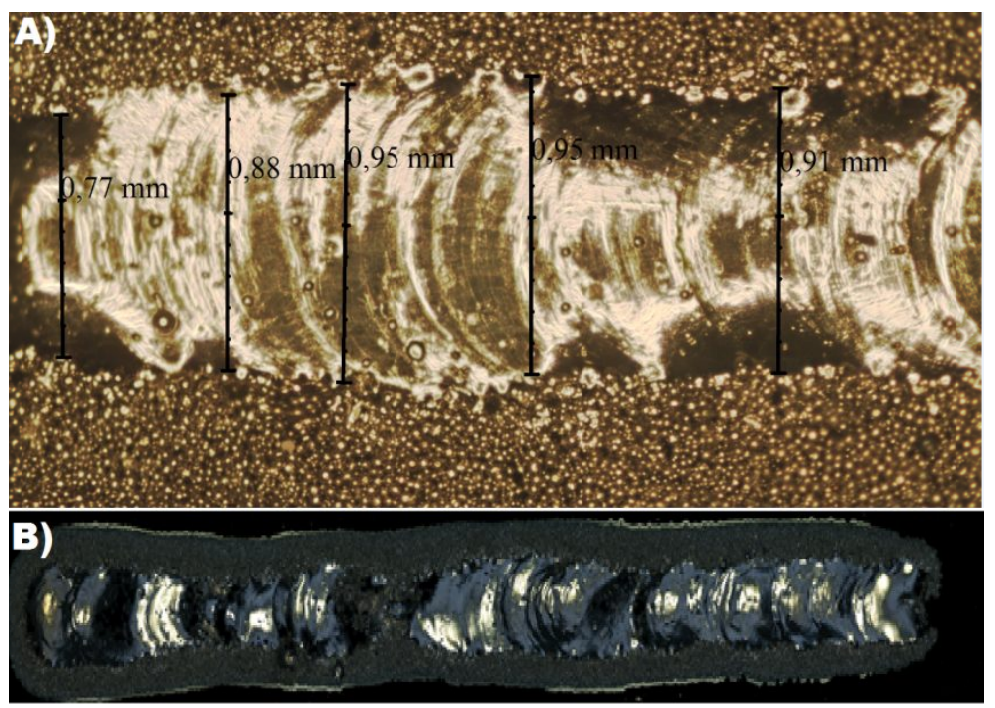

Figure 3 A) Molten tack width measured on single tracks formed by $50 \mathrm{~W}$ laser power, B) Single track formed by $120 \mathrm{~W}$ laser power showing molten and sintered zones 


\section{PHYSICAL MODEL DESCRIPTION}

In this study, the convection heat transfer in melt pool has been handled indirectly through effective material modelling. The laser material interaction has been introduced into the heat transfer model as a body heat source. The governing equation for the thermal phenomena during selective laser melting can then be given as

$$
\rho C_{p} \frac{\partial T}{\partial t}=\frac{\partial}{\partial x}\left(k_{x x} \frac{\partial T}{\partial x}\right)+\frac{\partial}{\partial y}\left(k_{y y} \frac{\partial T}{\partial y}\right)+\frac{\partial}{\partial z}\left(k_{z z} \frac{\partial T}{\partial z}\right)+\dddot{\Phi}
$$

where $\mathrm{T}$ is the temperature, $\mathrm{t}$ is the time, $(\mathrm{x}, \mathrm{y}, \mathrm{z})$ are the spatial co-ordinates, $\mathrm{k}_{\mathrm{xx}}, \mathrm{k}_{\mathrm{yy}}$ and $\mathrm{k}_{\mathrm{zz}}$ are the thermal conductivities in the different directions, $\rho$ is the density, $C_{p}$ is the specific heat and $\dddot{\Phi}$ is the heat source term. In the specific case of non-temperature dependent material properties, this equation reduces to

$$
\rho C_{p} \frac{\partial T}{\partial t}=k \nabla^{2} T+\dddot{\Phi}
$$

where $\mathrm{k}$ is the isotropic thermal conductivity. The thermal interaction at the boundaries between the material and the surroundings can be represented as

$$
-k \frac{\partial T}{\partial \eta}=-h\left(T_{a m b}-T\right)+\sigma \varepsilon\left(T^{4}-T_{a m b}^{4}\right)
$$

where $\mathrm{h}$ is the heat transfer coefficient between the material and the gaseous environment, $\mathrm{T}_{\mathrm{amb}}$ is the temperature of the gaseous environment, $\varepsilon$ is the emissivity of the material and $\sigma$ is the Stefan-Boltzmann constant.

\section{Laser Irradiation Models}

A typical approach when modelling the laser irradiation is by considering the radiative transfer equation [14] [15] [16] [17], which tries to capture the idea that the intensity of the laser beam entering into a given volume is attenuated due to absorption and scattering and simultaneously enhanced by secondary emissions by the materials inside the volume and the scattered energy entering from neighboring volumes. Mathematically, for a one-dimensional problem it is given by

$$
\mu \frac{\partial I(z, \mu)}{\partial \mu}=-(\alpha+\sigma) I(z, \mu)+\alpha I_{b}(z)+\frac{\sigma}{2} \int_{-1}^{1} \Phi(\Theta) I\left(z, \mu^{\prime}\right) d \mu^{\prime}
$$

where $z$ is the abscissa of the radiation intensity of $I$ of cosine direction $\mu$ with respect to $\mathrm{z}$ axis, $\Theta$ is the angle between an incoming radiation of cosine direction $\mu^{\prime}$ and the actual radiation intensity, $I_{b}$ is the blackbody radiation, $\alpha$ is the absorption coefficient, $\sigma$ is the scattering coefficient, and $\Phi$ is the scattering phase function. Often, it is easier to use extinction coefficient $\beta$ and scattering albedo $\omega$ instead of absorption and scattering coefficients. These properties are related as

$$
\begin{aligned}
\beta & =\alpha+\sigma \\
\omega & =\frac{\sigma}{\sigma+\alpha}
\end{aligned}
$$

Based on the independent scattering theory [18], the values for the extinction coefficient for a powder bed can be approximated by the following equations

$$
\boldsymbol{\beta}=\pi \boldsymbol{R}^{2} \boldsymbol{\eta}
$$

where $\eta$ is the number of particles per unit volume, $\beta$ is the extinction coefficient and $R$ is the average radius of the powder in the powder bed. Gusarov and Smurov [16] have adopted this approach in their work on modelling of single track formation with SLM.

However, simpler empirical models exist which utilize the extinction coefficient and scattering albedo to approximate an effective absorption coefficient for the powder bed, reducing the necessity to solve the radiative transfer equation. One such model based upon the assumption of diffusive reflections on powder particles, defines the effective powder bed absorptivity as

$$
A^{\text {effective }}=\frac{3}{4}\left(\frac{1-\omega+3 a}{1+2 a}\right)
$$




$$
a=\left(1-\frac{2}{3} \omega+\frac{1}{3} \omega^{2}\right)^{1 / 2}
$$

\section{Material modelling of powder beds}

The thermal properties of standard materials, based on experimental measurements, are typically available in literature. However, the values often correspond to bulk material properties, and cannot be directly applied to powder beds, which are characterized by discrete spatial distribution of materials. The typical approach is to substitute the discrete powder bed by a continuum of material possessing equivalent material properties. In this regard, Sih and Barlow [19] [20]have proposed successively improving predictive models for emissivity and thermal conductivity of powder beds. Shapiro et al [21]considered the powder bed as a network of thermal resistances connected at the contact points and via the gaseous medium, and proposed a theoretical model accounting for the size-dependence of the conductivity. Gusarov and Kovalev [22] have further developed the concept of the powder bed as a network of discrete thermal resistances and have proposed a dependency of effective thermal properties on the morphology of the powder bed.

In this study, the predictive model proposed by Sih and Barlow [23] for computing the effective material properties of the powder bed is adopted. The effective emissivity of the powder bed is described as a combination of the emissivity of the particles and the emissivity of the cavities in the powder bed.

$$
\varepsilon_{h}=A_{h} \varepsilon_{h}+\left(1-A_{h}\right) \varepsilon_{s}
$$

where $\varepsilon$ is the effective emissivity of the powder bed, $\varepsilon_{\mathrm{s}}$ is the emissivity of the bulk material, $\varepsilon_{\mathrm{h}}$ is the emissivity of the cavities and $A_{h}$ is the area fraction of surface occupied by the cavities. The area fraction $A_{h}$ is a function of the porosity $(\phi)$ of the randomly packed powder bed and is given as.

$$
A_{h}=\frac{0.908 \phi_{0}^{2}}{1.908 \phi_{0}^{2}-2 \phi_{0}+1}
$$

The emissivity of the cavities is also dependent on the porosity of powder bed and can then be estimated by

$$
\varepsilon_{h}=\frac{\varepsilon_{S}\left[2+3.082\left(\frac{1-\phi_{0}}{\phi_{0}}\right)^{2}\right]}{\varepsilon_{S}\left[1+3.082\left(\frac{1-\phi_{0}}{\phi_{0}}\right)^{2}\right]+1}
$$

In case of a randomly packed powder bed formed of mono-sized spherical powder particles, the effective thermal conductivity can be approximated by the modified Zehner-Schlünder-Damköhler equation

$$
\frac{k}{k_{f}}=\left(1-\sqrt{1-\phi_{0}}\right)\left(1+\phi_{0} \frac{k_{r}}{k_{f}}\right)+\sqrt{1-\phi_{0}}\left(\frac{2}{1-\frac{k_{f}}{k_{s}}}\left(\frac{1}{1-\frac{k_{f}}{k_{s}}} \ln \left(\frac{k_{s}}{k_{f}}\right)-1\right)+\frac{k_{r}}{k_{f}}\right)
$$

where $\mathrm{k}$ is the effective thermal conductivity of the powder bed, $\phi_{0}$ is the porosity of the powder bed, $\mathrm{k}_{\mathrm{s}}$ is the thermal conductivity of the bulk material, $\mathrm{k}_{\mathrm{f}}$ is the thermal conductivity of the gaseous environment, and $\mathrm{k}_{\mathrm{r}}$ is the equivalent thermal conductivity arising due to inter-particle radiation and is given by

$$
k_{r}=4 F \varepsilon \sigma_{B} T_{p}^{3} D_{p}
$$

where $\sigma_{\mathrm{B}}$ is the Stefan-Boltzman constant, $\mathrm{T}$ is the mean absolute temperature and $\mathrm{D}_{\mathrm{p}}$ is the diameter of powder particles. $\mathrm{F}$ is called the view factor, and can be chosen as a function of the emissivity of the powder bed leading to

$$
k_{r}=\frac{4 \varepsilon \sigma_{B} T_{p}^{3} D_{p}}{1-0.132 \varepsilon}
$$




\section{Implementation of 3D Finite Volume Alternate Direction Implicit Model}

The 3D finite volume alternate direction implicit model is a high-fidelity model developed for faster thermal calculations while still preserving accuracy (the accuracy is similar to Crank-Nicholson method). The theoretical basis can be found in [24] while the application of it in modelling selective laser melting can be found in [5].

\section{UNCERTAINTY CHARACTERIZATION OF SINGLE TRACK}

Monte Carlo simulation based uncertainty analysis techniques do not have an extensive requirement for experimental data. The Monte Carlo based methods can be used with an expert value of parameter uncertainty around the nominal values, both supplied by the user based on experience or drawn from maximum likelihood estimation studies for concerned parameters The accuracy of the predictions and the confidence intervals, however, are dependent on the manner of choosing perturbed parameter values for simulations, and thus any prior knowledge of the correlation between parameters lead to better results. The uncertainty analysis is first performed to calculate the probability distribution for melt track width and maximum domain temperature for the tracks formed with the nominal laser power of $50 \mathrm{~W}$, given an expert uniform uncertainty on seven input parameters - beam power, the beam FWHM, the porosity of the powder bed, the diameter of powder particles, the laser scan speed, the chamber temperature and the powder albedo (not to be confused with albedo of bulk material which is much lower). The procedure and results are found in [5]. It was suggested that once the uncertainty on parameters and outputs is established, the generated information such as correlation matrix can be used to perform uncertainty analysis at other parameter ranges.

\section{META-MODELLING OF SINGLE TRACK FORMATION}

The results of the Monte Carlo simulations can further be used to generate simpler regression models specific to each output, which can be used in place of physics-based models for faster albeit lower fidelity calculations. The accuracy of these regression models would be dependent on the quality of the Monte Carlo simulations, which in turn depend on the input uncertainty used. In this case, the two outputs of interest i.e. the width of melt track and the maximum domain temperature would require their own linear regression models. The standard regression coefficients (SRCs) for the two regression models are shown in Table 1. The absolute values of the SRCs correspond to the measure of influence the parameter has on the particular output, and can be sorted to generate the parameter significance ranking

Table 1 Standard regression coefficients for regression models predicting width of melt track and maximum domain temperature

\begin{tabular}{ccc}
\hline Parameter & $\begin{array}{c}\text { Standard Regression Coefficients for } \\
\text { width of melt track }\end{array}$ & $\begin{array}{c}\text { Standard Regression Coefficients for } \\
\text { maximum domain temperature }\end{array}$ \\
\hline Power & 0.0651 & 0.0950 \\
FWHM & -0.0515 & -0.4106 \\
Porosity & 0.0329 & 0.0860 \\
Powder Diameter & -0.4799 & -0.4351 \\
Scan Speed & 0.0340 & 0.0047 \\
Chamber Temperature & 0.0573 & 0.0688 \\
Powder Albedo & -0.9210 & -0.8827 \\
\hline
\end{tabular}



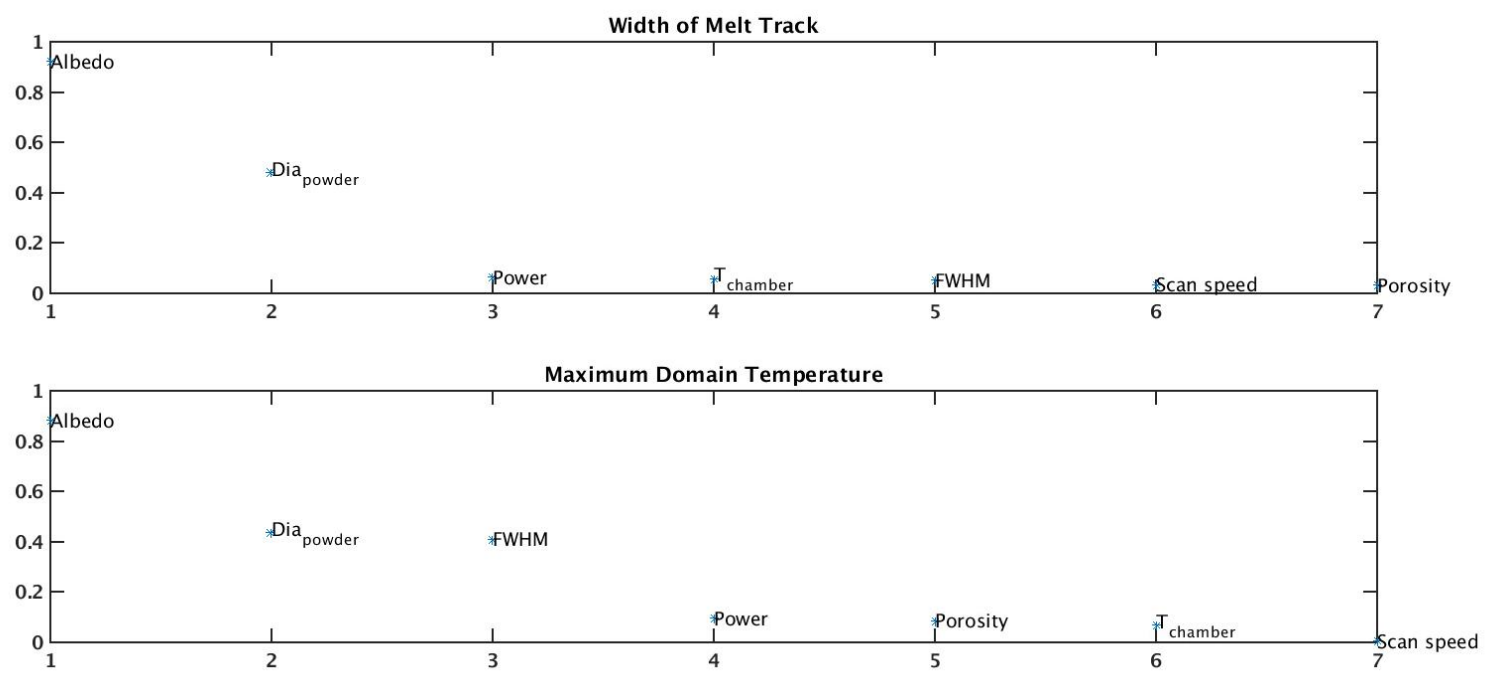

Figure 4 Parameter significance rankings for the two linear regression models corresponding to width of melt track (top) and maximum domain temperature (bottom)

The parameter significance ranking can be graphical represented together with the SRC as shown in Figure 4. Determination of SRCs, however, is not sufficient for accepting a particular model, especially when the original model is non-linear as in this case - the $\mathrm{R}^{2}$ value, which is a measure of the goodness of fit, for the regression model also has to be verified. Figure 5 shows the $\mathrm{R}^{2}$ value for the regression model corresponding to maximum domain temperature. In the figure, the blue dots correspond to values of maximum domain temperature for the parameter subsets used in the Monte Carlo simulations. The $\mathrm{x}$ - and $\mathrm{y}$ - axis correspond to the value from the regression model and the Monte Carlo simulation respectively, shown on a scale normalized with respect to the standard deviation. A high $\mathrm{R}^{2}$ value, as in this case, implies an acceptably accurate linear regression model and the value of 0.9598 implies that the linear regression model is able to account for $95.98 \%$ of the variance in maximum domain temperatures observed in the Monte Carlo simulation results.

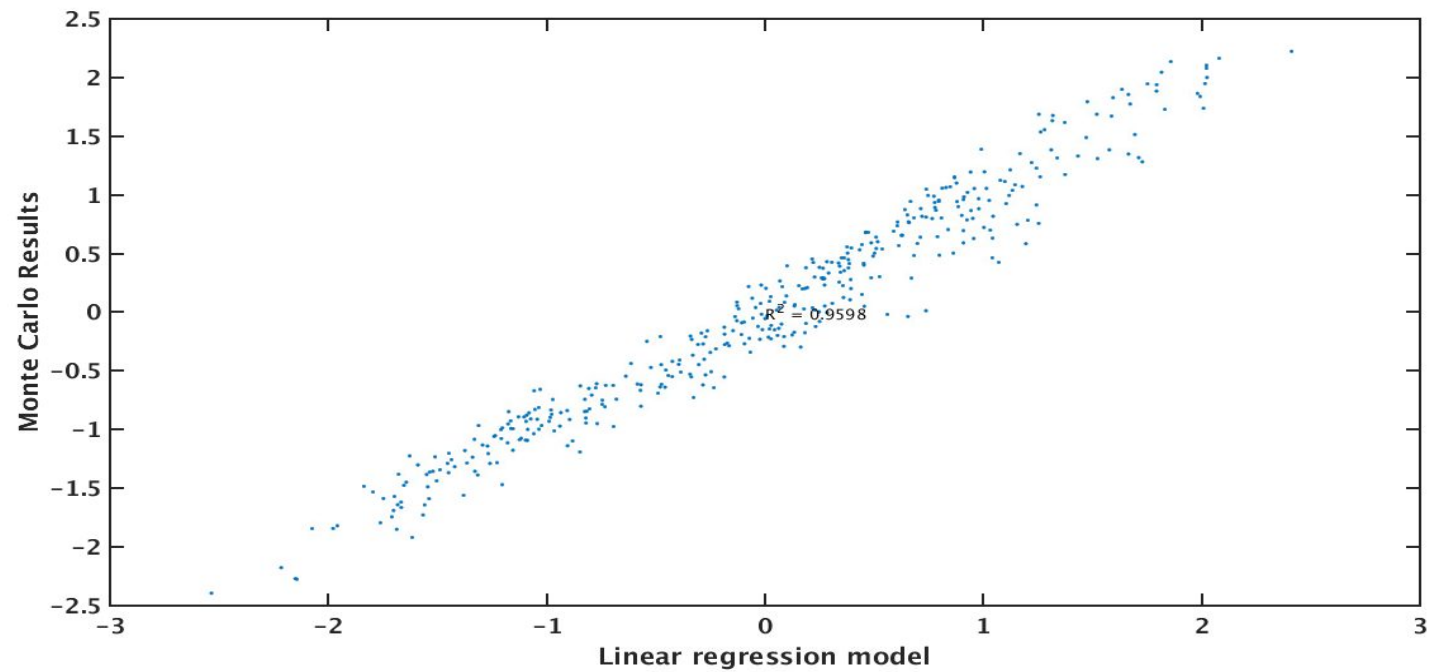

Figure 5 Coefficient of determination for linear regression model of SLM for maximum domain temperature 


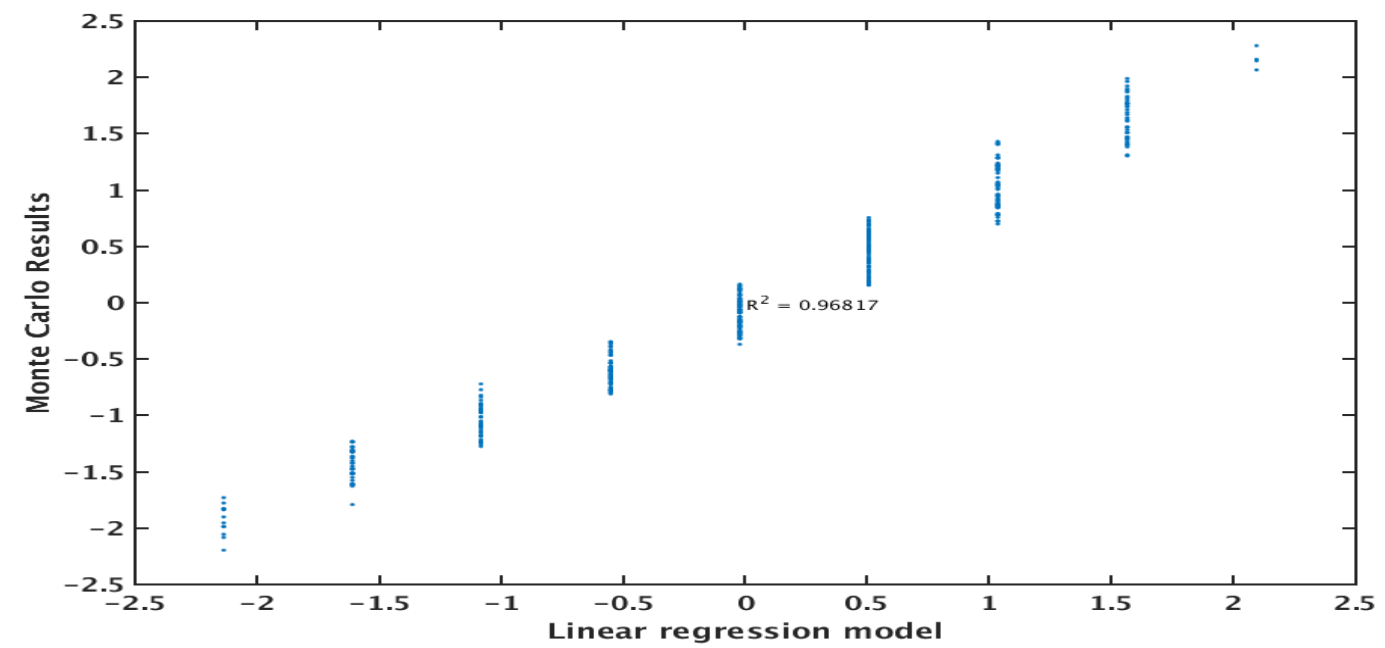

Figure 6 Coefficient of determination for linear regression model of SLM for width of melt track

Figure 6 shows the coefficient of determination $\left(\mathrm{R}^{2}\right)$ for the regression model corresponding to the width of melt track. The observed $R^{2}$ value of 0.96817 is high enough for accepting the regression model as a good predictor model within the selected range of parameters. The clustering of the predicted values, as observed in the figure, is due to the spatial resolution of the current model which in turn is an outcome of the spatial discretization used.

The regression models developed can be used in lieu of physics-based models as response surface generators. As a validation, the regression model developed using the uncertainty analysis results from the $50 \mathrm{~W}$ laser power melt tracks is used to predict the melt track width at $120 \mathrm{~W}$ laser power while all other parameters remain constant. The predicted value of melt track widths generated through the regression model for $120 \mathrm{~W}$ laser power is compared with the measured melt track widths and is found to have a good match. Figure 7 shows the measured and predicted melt track widths for the $120 \mathrm{~W}$ laser power with the associated standard deviations. In both cases, the distribution is centered around $2.65 \mathrm{~mm}$ with a standard deviation of around $0.5 \mathrm{~mm}$.
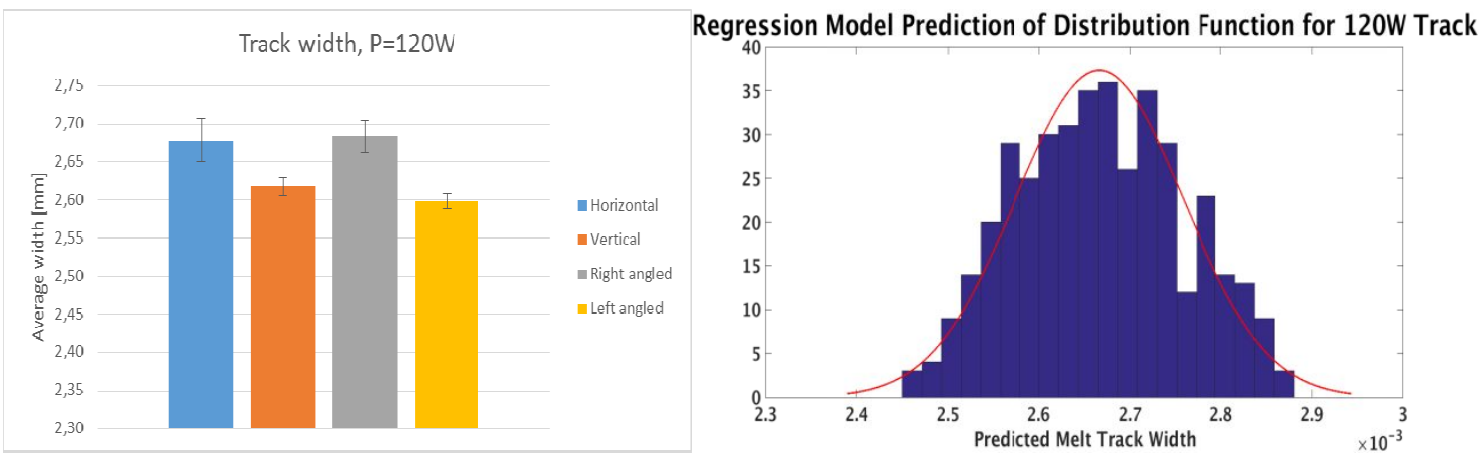

Figure 7 Measured melt track width of single tracks generated in four directions for laser power of $120 \mathrm{~W}$ (left) and predicted distribution function for melt track width from regression model (right) 


\section{UNCERTAINTY CHARACTERIZATION OF SINGLE LAYER FORMATION}

Calibration studies on single melt tracks suggest that small perturbations in the laser related parameters such as power, beam FWHM and the corresponding absorptivity of the material had a greater effect on the track dimensions than other parameters considered in the study. The effect of processing chamber temperature on the properties of the parts made by SLM however is well-documented in literature. Moreover, these four parameters had been chosen for uncertainty analysis of single layer production using six different scanning strategies [25] [26] using expert uncertainties. However, the information/process knowledge obtained during uncertainty analysis of the single tracks can be transferred as input to the uncertainty analysis of single layer formation.

In this implementation, the uniform expert input uncertainty is replaced by a probability distribution for each parameter about the nominal value. The Latin Hypercube sampling results for this implementation is shown in Figure 8. As before, the uncertainty analysis was performed for the different scanning strategies with the percentage of area consolidated, the thermal homogeneity (standard deviation of temperature) and the maximum temperature as the outputs.

The nominal values of the parameters used for the uncertainty analysis are given in Table 2. However, the actual input values used for the analysis and the associated uncertainty values are taken from the parameter estimation with single tracks.

Table 2 Nominal values of parameters used for uncertainty analysis

\begin{tabular}{ll}
\hline Parameter & Nominal Values \\
\hline Power & $120 \mathrm{~W}$ \\
FWHM & $37.5 \mathrm{e}-6 \mathrm{~m}$ \\
Chamber Temperature & $27 \mathrm{C}$ \\
Powder Albedo & 0.9 \\
\hline
\end{tabular}

The 400 parameter sets generated by the sampling technique are then subjected to Monte Carlo simulations for each of the six scanning strategies. The obtained values of percentage of area consolidated, standard deviation of temperature and maximum domain temperature are then represented via cumulative probability distribution functions (CDF). The results for the six scanning strategies are shown in Figure 9 through Figure 14.

For each scanning strategy, the plots show the cumulative probability distribution for the percentage of consolidated area (left), maximum domain temperature(center) and the standard deviation of temperature at the end of scan path(right). As these are cumulative distribution curves, the steeper the slope of the curve, the more precise is the range of the corresponding output. The smaller range and distribution information, as captured by the sampling technique, lead to better prediction of output uncertainties. 


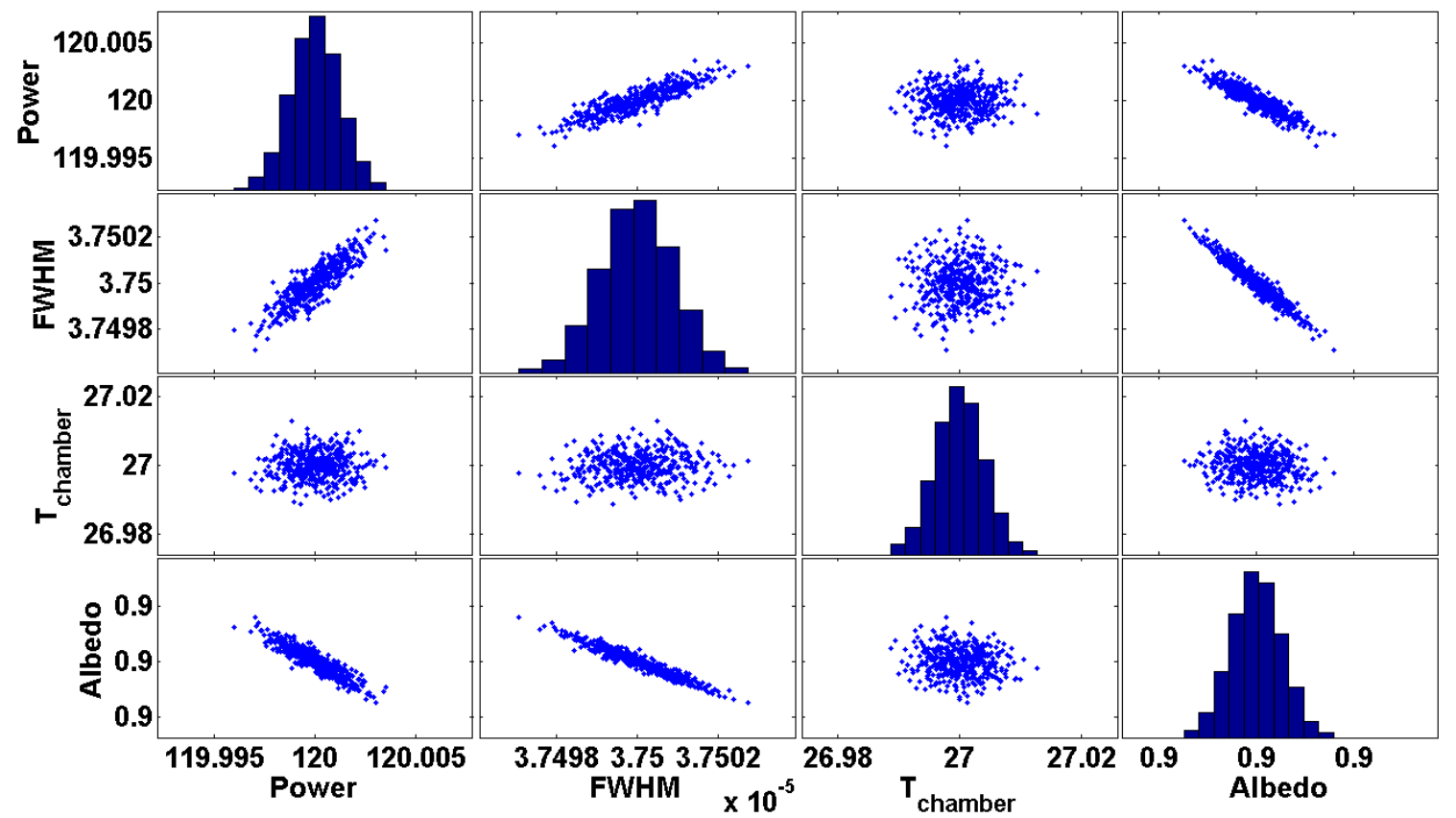

Figure 8 Latin hypercube sampling of parameter space for usage in uncertainty analysis
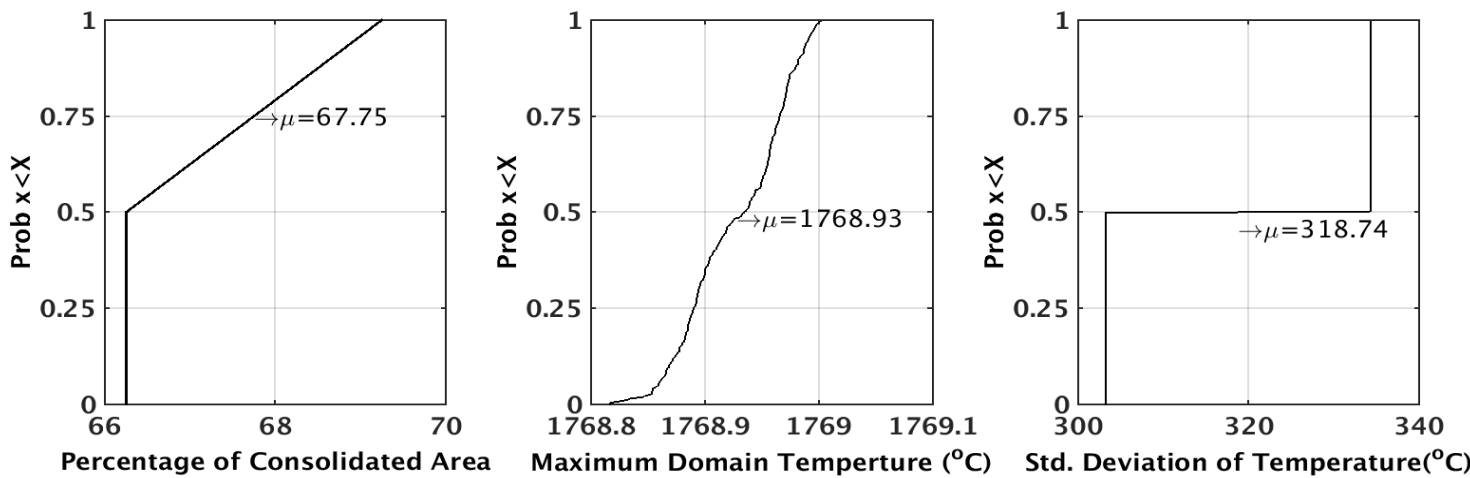

Figure 9 Cumulative probability distribution functions of percentage of consolidated area, maximum domain temperature and standard deviation of temperature for antiparallel/zigzag scanning path
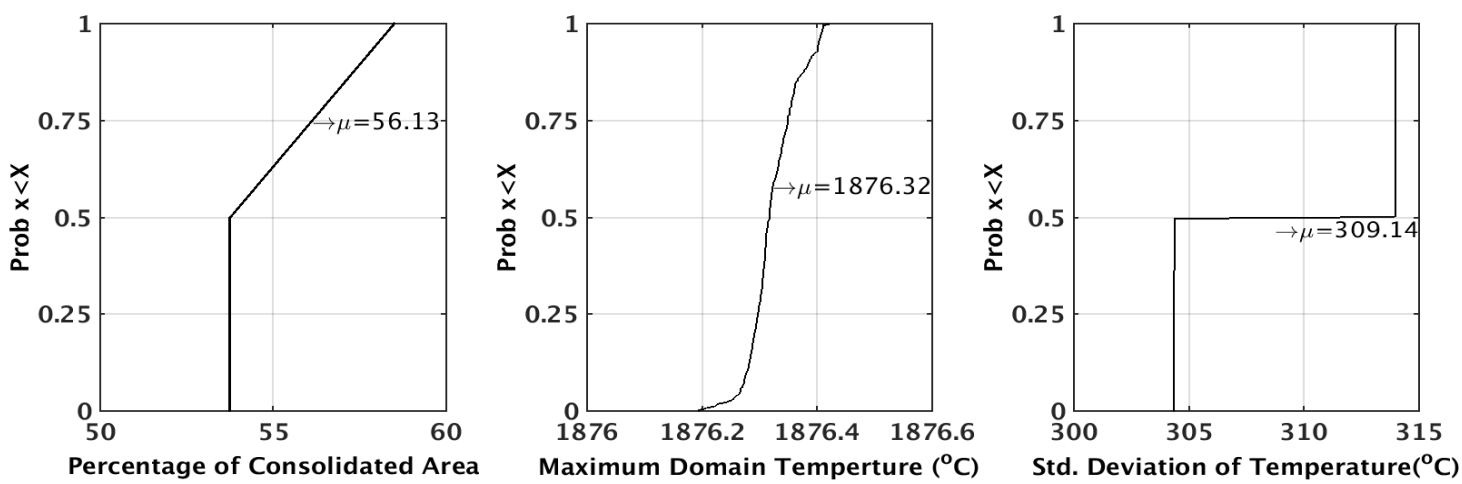

Figure 10 Cumulative probability distribution functions of percentage of consolidated area, maximum domain temperature and standard deviation of temperature for inspiral scanning path 

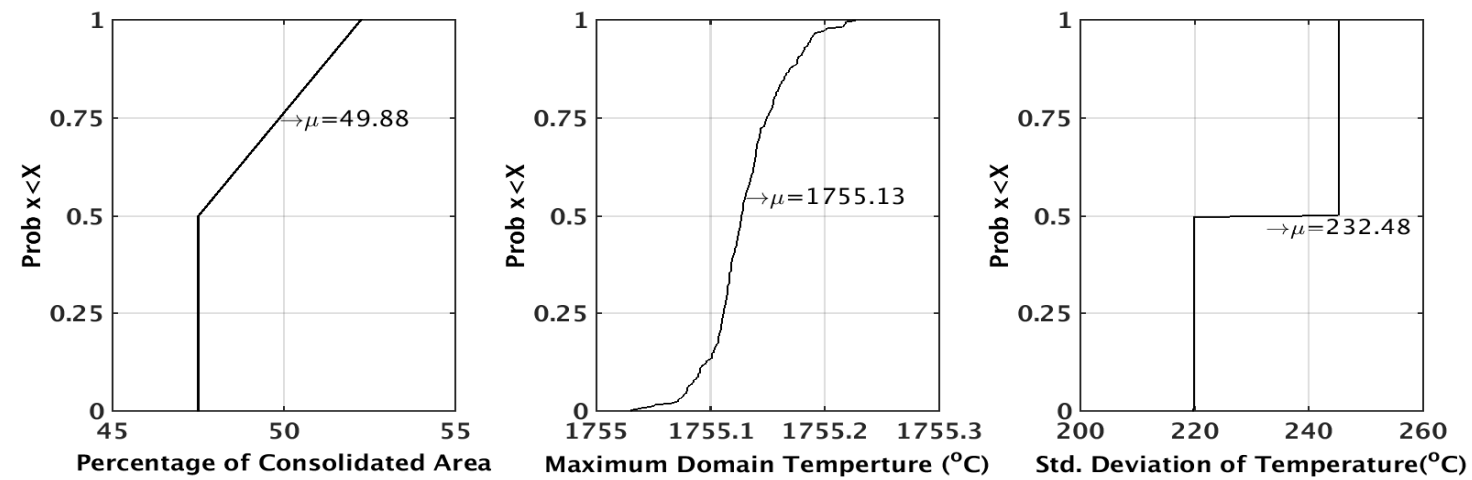

Figure 11 Cumulative probability distribution functions of percentage of consolidated area, maximum domain temperature and standard deviation of temperature for outspiral scanning path
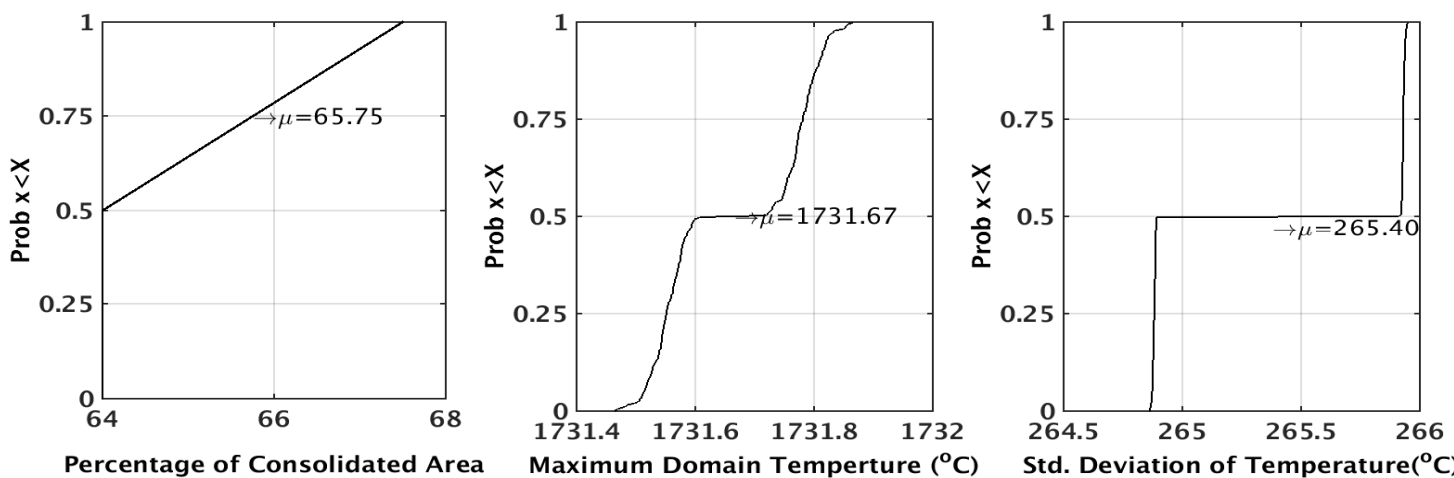

Figure 12 Cumulative probability distribution functions of percentage of consolidated area, maximum domain temperature and standard deviation of temperature for parallel scanning path
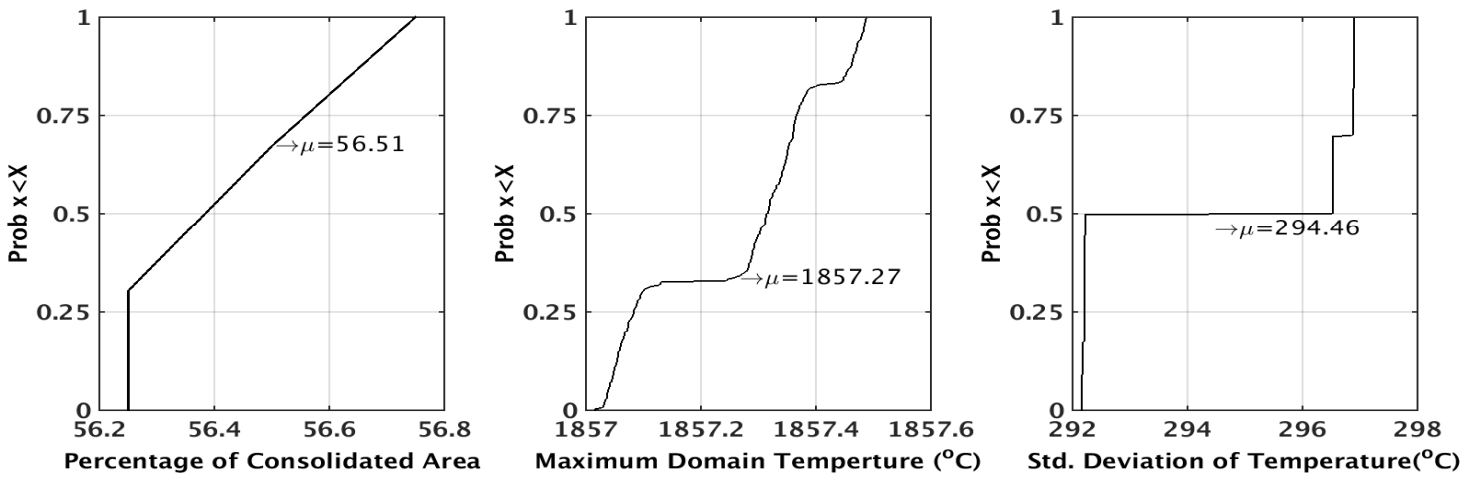

Figure 13 Cumulative probability distribution functions of percentage of consolidated area, maximum domain temperature and standard deviation of temperature for parallel interlaced scanning path 

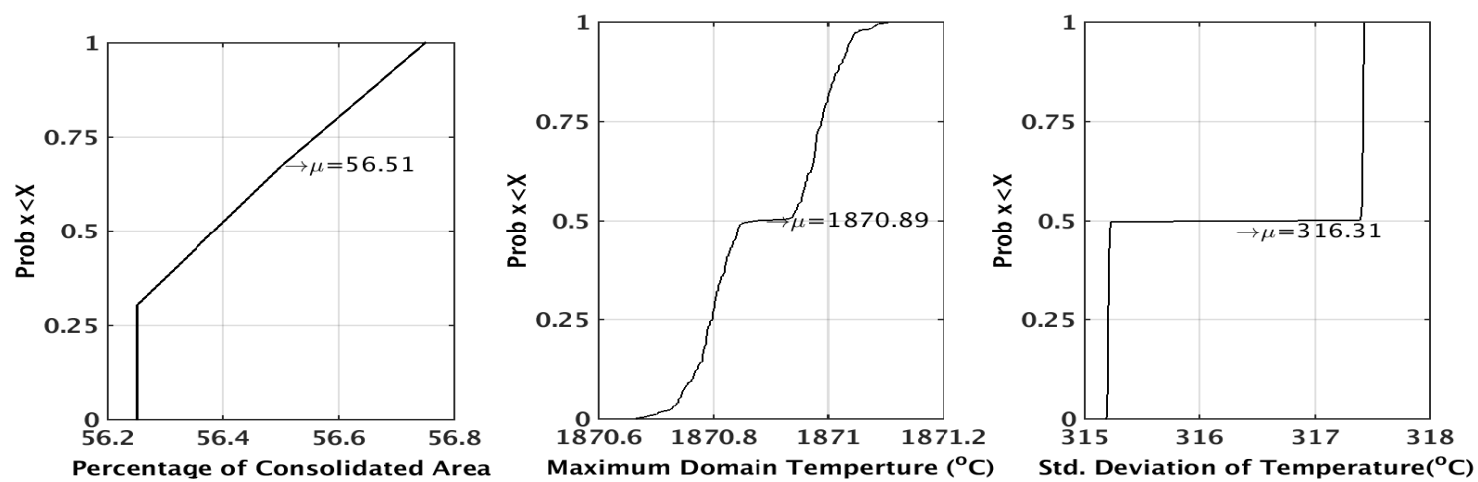

Figure 14 Cumulative probability distribution functions of percentage of consolidated area, maximum domain temperature and standard deviation of temperature for parallel interlaced reverse scanning path

When using non-uniform input uncertainty, the CDFs for the three outputs are observed to be steeper and within a smaller range. This implies a lower uncertainty in outputs is predicted when using probability distributions on input parameters (experimentally-derived or expert) as opposed to uniform selection over the entire range. Moreover, the current uncertainty analysis results are much more useful when comparing between scan strategies as opposed to the previous implementation e.g. the antiparallel and parallel scanning strategies are easily seen to be better than other strategies with respect to percentage of consolidated areas. Intuitively, the usage of probability distributions in association with input parameters has a greater correspondence to real processing where parameters are more likely to be closer to the designed/prescribed value than farther away.

\section{CONCLUSION}

In this paper, a systematic approach towards establishing reliability of overhanging structure production by selective laser melting has been adopted. Single tracks are first manufactured on a deep powder bed using chosen processing parameters, but at different locations in the powder bed and in different laser scanning directions. The experimental results for the $50 \mathrm{~W}$ laser power are used in combination with numerical model, and subjected to uncertainty and reliability analysis. Based on the uncertainty characterization results, a regression model is developed which is deemed to be capable of use as a response surface generator. Subsequently, the regression model is validated using the $120 \mathrm{~W}$ laser power melt track.

Thereafter, the technique is extended for reliability characterization of single layers produced on a thick powder bed without support structures, by determining cumulative probability distribution functions for outputs corresponding to maximum recorded temperature, sample density and thermal homogeneity.

Parametric study results for common SLM parameters are available in literature and, in the future, it is of interest to couple this global knowledge with the local knowledge obtained using the uncertainty analysis techniques used in this paper.

\section{ACKNOWLEDGEMENT}

The authors of the paper acknowledge the contributions of the FMAT consortium and the Danish Technological Institute which provided the facilities for manufacturing the optimized scanning strategies. 


\section{REFERENCE}

[1] O. Rehme, Cellular design for laser freeform fabrication, $\mathrm{PhD}$ Thesis, Technical University Hamburg, 2009.

[2] S. Clijsters, T. Craeghs, S. Buls, K. Kempen and J. Kruth, "In situ quality control of the selective laser melting process using a high speed, real-time melt pool monitoring system," The International Journal of Advanced Manufacturing Technology, vol. 75, no. 5-8, pp. 1089-1101, 2014.

[3] H. Rieder, A. DillHofer, M. Spies, J. Bamberg and T. Hess, "Online monitoring of additive manufacturing processes using ultrasound," in ECNDT 2014, Prague, 2014.

[4] I. Yadroitsev and I. Smurov, "Factor analysis of selective laser melting process parameters and geometrical characteristics of synthesized single tracks," Rapid Prototyping Journal, pp. 201-208, 2012.

[5] S. Mohanty and J. Hattel, "Numerical Model based Reliability Estimation of Selective Laser Melting Process," Physics Proceedia, vol. 56, pp. 379-389, 2014.

[6] S. A. Khairallah and A. Anderson, "Mesoscopic simulation model of selective laser melting of stainless steel powder," Journal of Materials Processing Technology, vol. 214, pp. 2627-2636, 2014.

[7] K. Dai and X. Li, "Comparison between thermal modeling and experiments: Effects of substrate preheating," Rapid Prototyping Journal, pp. 24-34, 2004.

[8] F. B. and L. Mullen, "Gas flow effects on selective laser melting manufacturing performance," Journal of Materials Processing Technology, pp. 355-364, 2012.

[9] J. Guo and X. Du, "Reliability analysis for multidisciplinary systems with random and internal variables," AIAA, vol. 48, no. 1, pp. 82-91, 2010.

[10] A. De and D. T, "Reliable calculations of heat and fluid flow during conduction mode laser welding through optimization of uncertain parameters," Welding Journal, vol. 38, pp. 2977-85, 2005.

[11] C. Abreu and F. Miranda, "Sensitivity analysis of laser cutting based on metamodeling approach," in Handbook of research on computational simulation and modeling in engineering, IGI Global, 2015.

[12] A. Saltelli, M. Ratto, S. Tarantola and F. Camppolongo, "Sensitivity analysis practices : Strategies for model based inference," Reliability Engineering and Systems Safety, vol. 91, pp. 1109-1125, 2006.

[13] M. Saisana, A. Saltelli and S. Tarantola, "Uncertainty analysis and sensitivity analysis techniques as tools for the quality assessment of composite indicators," Journal of the Royal Statistical Society-A, vol. 168, no. 2, pp. 307-323, 2005.

[14] A. Gusarov, "Radiation transfer in metallic powder beds during laser forming," Quantum Electronics, pp. 451-459, 2010.

[15] A. Gusarov and J. Kruth, "Modelling of radiation transfer in metallic powders at laser treatment," International Journal of Heat and Mass Transfer, pp. 3423-3434, 2005.

[16] A. Gusarov and I. Smurov, "Modelling the interaction of laser radiation with powder bed at selective laser melting," Physics Proceedia, pp. 381-394, 2010.

[17] A. Gusarov and I. Smurov, "Radiation transfer in metallic powdwer beds in laser processing," Journal of Quantitative Spectroscopy \& Radiative Transfer, pp. 2571-2527, 2010. 
[18] B. Drolen and C. Tien, "Independent and dependent scattering in packed sphere system," Journal of Thermophysics and Heat Transfer, pp. 63-68, 1987.

[19] S. Sih and J. Barlow, "The prediction of the thermal conductivity of powders," in Proceedings of the 6th Annual International Solid Freeform Fabrication Symposium, Austin, USA, 1995.

[20] S. Sih and J. Barlow, "Emissivity of powder beds," in Proceedings of the 6th Annual International Solid Freeform Fabrication Symposium, Austin, USA, 1995.

[21] M. Shapiro, V. Dudko, V. Royzen, Y. Krichevets, S. Lekhtmaker, S. Grozubinsky and M. Brill, "Characterization of powder beds by thermal conductivity: Effect of gas pressure on the thermal resistance of particle contact points," Part. Part. Syst. Charact., vol. 21, pp. 268-275, 2004.

[22] A. Gusarov and E. Kovalev, "Model of thermal conductivity in powder beds," Physical Review B, vol. 80, no. 2, p. 024202, 2009.

[23] S. Sih and J. Barlow, "The prediction of the emissivity and thermal conductivity of powder beds," Particulate Science and Technology, vol. 14, no. 3, pp. 291-304, 2004.

[24] S. Mohanty and J. Hattel, "A finite volume volume alternate direction implicit approach to modelling selective laser melting," in Proceedings of 32nd International Congress on Applications of Lasers and Electro-optics, 2014.

[25] S. Mohanty, C. Tutum and J. Hattel, "Cellular scanning strategy for selective laser melting: evolution of optimal grid-based scanning path and parametric approach to thermal homogeneity," in Proceedings of SPIE 8608, Laser-based Micro- and Nano-packaging and Assembly VII, San Francisco, USA, 2013.

[26] S. Mohanty and J. Hattel, "Cellular scanning strategy for selective laser melting: Generating reliable, optimized scanning paths and processing parameters," Proceedings of SPIE: Laser 3D Manufacturing II, vol. 9353, p. 9353OU, 2015.

[27] R. Mertens, S. Clijsters, K. Kempen and J. Kruth, "Optimization of Scan Strategies in Selective Laser Melting of Aluminum Parts With Downfacing Areas," J. Manuf. Sci. Eng., vol. 136, no. 6, 2014.

[28] S. Mohanty and J. Hattel, "Cellular Scanning Strategy for Selective Laser Melting: Capturing Thermal Trends with a Low-Fidelity, Pseudo-Analytical Model," Mathematical Problems in Engineering, vol. 2014, no. Article ID 715058, p. 14 pages, 2014.

[29] M. Zaeh and B. G., "Investigations on residual stresses and deformations in selective laser melting," Production Engineering, vol. 4, no. 1, pp. 35-45, 2010.

[30] I. Yadroitsev and A. Gusarov, "Single track formation in selective laser melting of metal powders," Journal of Materials Processing Technology, pp. 1624-1631, 2010.

[31] F. Gürtler, M. Karga, K. Leitza and M. Schmidt, "Simulation of laser beam melting of steel powders using the three-dimensional volume of fluid method," Physics Proceesia, vol. 41, pp. 874-879, 2013.

[32] A. Gusarov and Y. I., "Heat transfer modelling and stability analysis of selective laser melting," Applied Surface Science, pp. 975-979, 2007. 